\title{
HIV latency is reversed by ACSS2-driven histone crotonylation
}

\author{
Guochun Jiang, ${ }^{1}$ Don Nguyen, ${ }^{1}$ Nancie M. Archin, ${ }^{2}$ Steven A. Yukl, ${ }^{2}$ Gema Méndez-Lagares, ${ }^{1}$ Yuyang Tang, ${ }^{1}$ Maher M. Elsheikh, ${ }^{1}$ \\ George R. Thompson III,' Dennis J. Hartigan-O'Connor, ${ }^{1}$ David M. Margolis, ${ }^{2}$ Joseph K. Wong, ${ }^{3}$ and Satya Dandekar'
}

'Department of Medical Microbiology and Immunology, UCD, Davis, California, USA. 'Department of Medicine, University of North Carolina at Chapel Hill, Chapel Hill, North Carolina, USA. ${ }^{3}$ Department of Medicine, UCSF, and San Francisco Veterans Affairs Medical Center, San Francisco, California, USA.

\begin{abstract}
Eradication of HIV-1 (HIV) is hindered by stable viral reservoirs. Viral latency is epigenetically regulated. While the effects of histone acetylation and methylation at the HIV long-terminal repeat (LTR) have been described, our knowledge of the proviral epigenetic landscape is incomplete. We report that a previously unrecognized epigenetic modification of the HIV LTR, histone crotonylation, is a regulator of HIV latency. Reactivation of latent HIV was achieved following the induction of histone crotonylation through increased expression of the crotonyl-CoA-producing enzyme acyl-CoA synthetase short-chain family member 2 (ACSS2). This reprogrammed the local chromatin at the HIV LTR through increased histone acetylation and reduced histone methylation. Pharmacologic inhibition or siRNA knockdown of ACSS2 diminished histone crotonylation-induced HIV replication and reactivation. ACSS2 induction was highly synergistic in combination with either a protein kinase $C$ agonist (PEP005) or a histone deacetylase inhibitor (vorinostat) in reactivating latent HIV. In the SIV-infected nonhuman primate model of AIDS, the expression of ACSS2 was significantly induced in intestinal mucosa in vivo, which correlated with altered fatty acid metabolism. Our study links the HIV/SIV infection-induced fatty acid enzyme ACSS2 to HIV latency and identifies histone lysine crotonylation as a novel epigenetic regulator for HIV transcription that can be targeted for HIV eradication.
\end{abstract}

\section{Introduction}

Immune cells harboring transcriptionally silent HIV-1 (HIV) are not detected by the host immune system in HIV-infected individuals with suppressive antiretroviral therapy (ART) and pose a major barrier to HIV eradication (1-6). HIV transcription is highly regulated and is responsive to several cell signaling pathways. Transcriptional activation of latent HIV has been reported using latency reversal agents (LRAs) that activate protein kinase C/ $\mathrm{NF}-\kappa \mathrm{B}$ and $\mathrm{pTEFb}$ signaling (7). It has been well recognized that epigenetic regulation of histone tails at the HIV long-terminal repeat (LTR) is critical for the establishment of latent reservoirs (8). Recent studies showed that inhibition of histone deacetylase (HDAC) or histone methyltransferase can reactivate HIV, and some of these inhibitors have been investigated as LRAs in human clinical trials (9-14). While HDAC inhibitors can reactivate latent HIV, their potency is low compared with those of other LRAs (7, 15, 16). The landscape of epigenetic modifications at the HIV LTR regulating viral transcription or latency is not fully defined. Additional unknown epigenetic modifications may exist that limit efficient HIV reactivation. Elucidation of these novel epigenetic modifications will provide a better understanding of viral transcriptional regulation and identify novel targets for drug discovery.

A recently discovered histone posttranslational modification by lysine crotonylation is involved in regulating host gene expres-

Conflict of interest: The authors have declared that no conflict of interest exists. Submitted: October 13, 2017; Accepted: January 9, 2018.

Reference information: / Clin Invest. 2018;128(3):1190-1198.

https://doi.org/10.1172/JCI98071. sion $(17,18)$. Histone crotonylation at the gene promoter can be induced by increasing of intracellular levels of crotonyl-CoA through the addition of sodium crotonate (Na-Cro) to cells (18, 19). However, it is not known whether histone crotonylation is involved in HIV transcription and whether it interacts with or influences other histone modifications at the HIV LTR that may be important for efficient HIV transcription. Similar to histone acetylation marks, several "writers" of histone crotonylation have been reported (20-22), including the crotonyl-CoA-producing enzyme acyl-CoA synthetase short-chain family member 2 (ACSS2).

The gastrointestinal tract, where the ACSS2 enzyme is an essential component of fatty acid metabolism, plays an important role in lipid homeostasis (23). It has been reported that HIV infection leads to lipid dysregulation (24-26), and we and others have reported that lipid metabolism was altered or dysregulated in HIV-infected primary CD4 ${ }^{+} \mathrm{T}$ cells as well as in intestinal tissues from individuals with early HIV infection (27-29). Interestingly, aberrant fatty acid metabolism is associated with immune dysregulation and nutritional complications in HIV patients with advanced disease (30-32). However, linkage between the changes in the fatty acid metabolism encompassing ACSS2 expression and HIV transcription and latency during the course of HIV disease has not been well investigated.

In this report, we demonstrate that histone crotonylation at the HIV LTR regulates HIV transcription and is involved in the establishment of HIV latency. The ACSS2 enzyme of fatty acid metabolism promotes histone crotonylation at the HIV LTR, leading to the reactivation of latent HIV and viral transcription in vitro and ex vivo, while suppression of ACSS2 inhibits HIV replication 

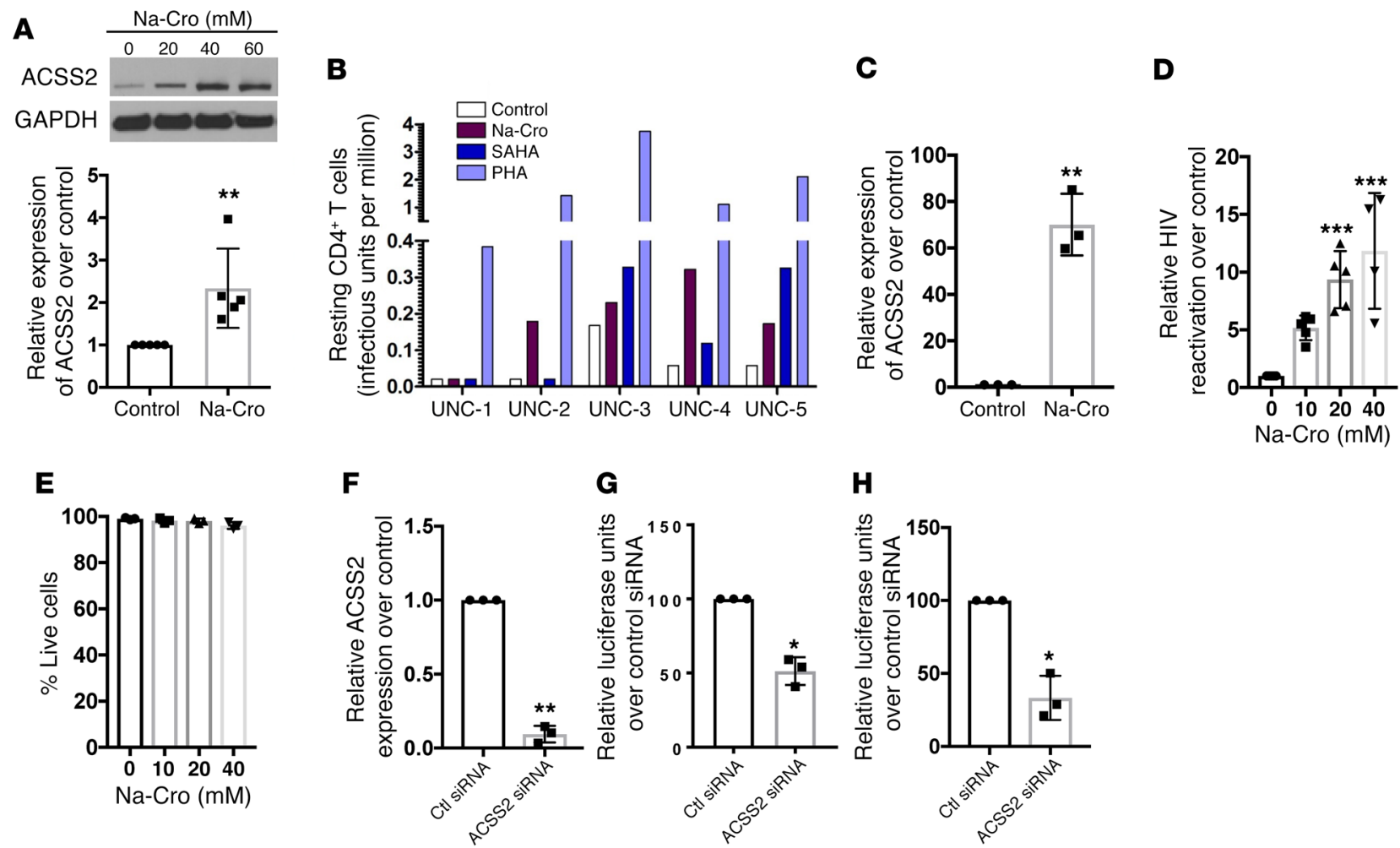

Figure 1. Induction of ACSS2 reactivates HIV from latency in vitro and ex vivo. (A) Expression of ACSS2 was induced in Na-Cro-treated primary CD4+ ${ }^{+}$cells from HIV-negative healthy donors $(n=5)$. Levels of ACSS2 protein (upper panel) or mRNA (lower panel) were induced in the primary CD4 ${ }^{+} T$ cells after incubation with Na-Cro for 6 hours. ${ }^{*} P<0.01$ vs. control treatment. (B) Induction of ACSS2 reactivated latent HIV in the resting CD4 ${ }^{+}$T cells from HIV-infected individuals $(n=5)$ under suppressive ART. Virus expression was compared using quantitative viral outgrowth assay in patients after the resting CD4 ${ }^{+} \mathrm{T}$ cells were treated with IL-2, IL-2 plus PHA, IL-2 plus vorinostat, or IL-2 plus Na-Cro. IUPM, infected units per million. (C) Na-Cro (30 mM) induced the expression of ACSS2 enzyme in J-Lat A1 cells after 4-hour incubation. ${ }^{* *} P<0.01$ vs. control treatment $(n=3)$. (D) Induction of ACSS2 by Na-Cro reactivated HIV transcription in a dose-dependent manner in J-Lat A1 cells as measured by quantitative reverse transcriptase PCR (RT-qPCR). ${ }^{* * *} P<0.001$ vs. control treatment $(n=$ 4). (E) Cellular toxicity was evaluated by flow cytometry after live/dead staining $(n=4)$. (F) Expression of ACSS2 was suppressed following siRNA knockdown of ACSS2. Expression of ACSS2 was measured by real-time PCR following transfection of ACSS2 siRNA into TZM-bl reporter cells. ${ }^{* *} P<0.01$ versus control treatment. (G and $\mathbf{H})$ Knocking down ACSS2 suppressed HIV replication in the TZM-bl reporter cell line using luciferase reporter assay without (C) or with (H) $\mathrm{Na}$-Cro treatment. ${ }^{*} P<0.05$ versus control siRNA knockdown $(n=3)$. The data were analyzed with a Student's $t$ test or 1-way ANOVA.

and reactivation of latent HIV. Our data suggest that ACSS2induced histone crotonylation is a novel epigenetic mark regulating viral transcription and may be an attractive target for developing new strategies for HIV eradication.

\section{Results}

Induction of histone crotonylation reactivates HIV from latency. Inducing crotonylation at histone tails in target genes by the addition of Na-Cro resulted in transcriptional activation (18). We sought to examine the effects of histone crotonylation in primary human $\mathrm{CD}^{+} \mathrm{T}$ cells. Expression of the crotonyl-CoA-converting enzyme ACSS2, at both the protein and the mRNA level, was induced in the primary $\mathrm{CD} 4^{+} \mathrm{T}$ cells from healthy HIV-negative donors following the addition of Na-Cro to the cultures (Figure 1A). To examine the effect of ACSS2 induction on HIV reservoirs, resting $\mathrm{CD} 4^{+} \mathrm{T}$ cells were isolated from peripheral blood of HIV-infected individuals $(n=5)$ with undetectable plasma viral loads (Supplemental Table 1; supplemental material available online with this article; https:// doi.org/10.1172/JCI98071DS1). Replication-competent HIV was recovered from the cells of 4 of the 5 HIV-infected donors following exposure to $\mathrm{Na}$-Cro, suggesting that induction of ACSS2 sup- ported the reversal of HIV latency ex vivo (Figure 1B). Interestingly, cells from the first donor (UNC-1) were not responsive to either SAHA (vorinostat) or Na-Cro, maybe because of a relatively lower proviral reservoir in comparison with the other patients.

Since the analysis of epigenetic marks from relatively a very small number of latently HIV-infected cells in vivo is challenging, we expanded the analysis of histone crotonylation for latent HIV reactivation to 2 well-characterized cell line models of HIV latency, J-Lat A1 cells (derived from Jurkat cells harboring 1 copy of HIV LTR-Tat-GFP gene) and U1 cells (a U937 promonocytic cell line harboring HIV genome with defective Tat gene). Similar to our findings in primary $\mathrm{CD}^{+} \mathrm{T}$ cells, addition of $\mathrm{Na}$-Cro to J-Lat A1 cells resulted in 70-fold increased expression of ACSS2 (Figure 1C). HIV LTR-driven viral transcription was induced in a dose-dependent manner, and Na-Cro increased HIV transcription 9- to 12-fold in comparison with untreated controls (Figure 1D). Minimal cellular toxicity was observed with concentrations of Na-Cro ranging from 10 to $40 \mathrm{mM}$ (Figure 1E). Similar effects of crotonylation were observed in the U1 latent HIV cell line model (Supplemental Figure 1). To determine the role of ACSS2 in the replication of HIV, we used ACSS2-specific siRNA to suppress the 
A
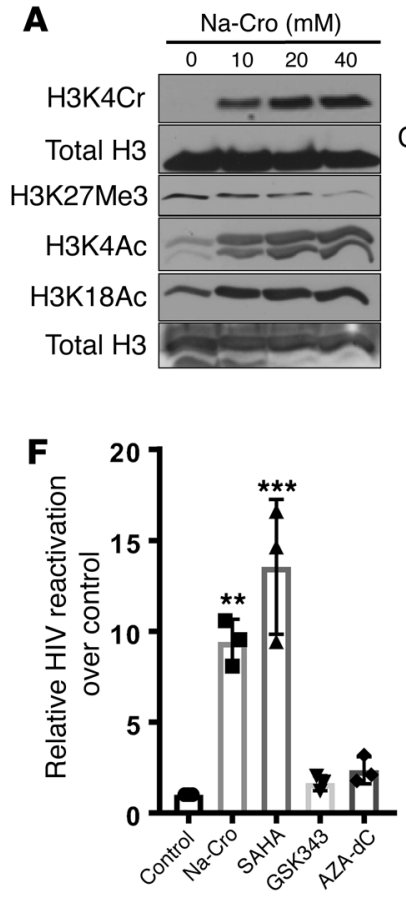

B

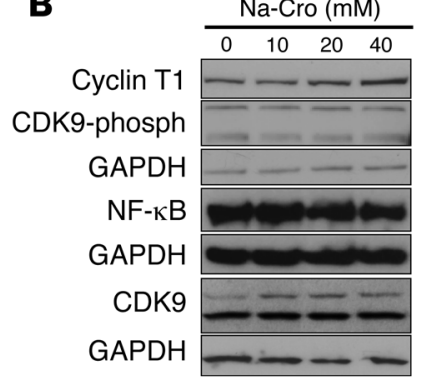

G

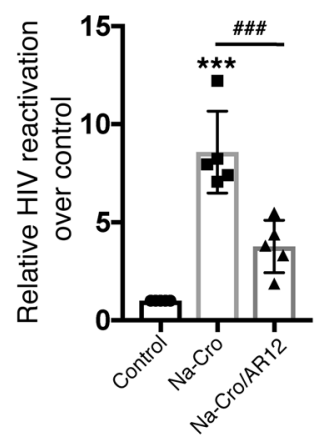

C

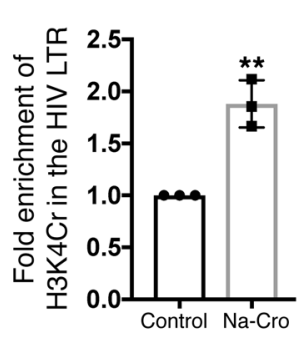

D

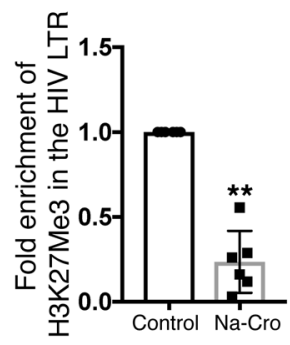

E

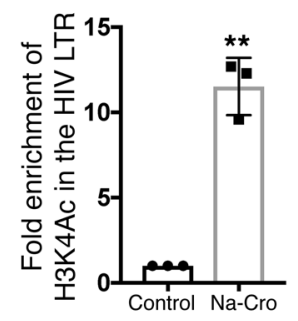

H

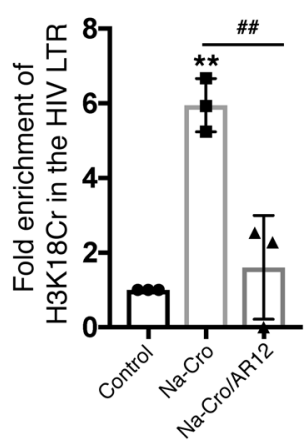

Figure 2. Histone crotonylation by ACSS2 induction reprograms histone tails at the HIV LTR, and suppression of ACSS2 dampens reactivation of latent HIV. (A) Induction of ACSS2 increased global expression of H3K4 crotonylation (H3K4Cr), H3K4 acetylation (H3K4Ac), and H3K18 acetylation (H3K18Ac) while decreasing expression of H3K27 methylation (H3K27Me) after Na-Cro treatment for 4 hours in J-Lat A1 cells. Immunoblots are representative of 3 independent experiments. (B) Protein expression after Na-Cro treatment for 4 hours in J-Lat A1 cells. Immunoblots are representative of 3 independent experiments. (C-E) Na-Cro addition induced histone crotonylation at $\mathrm{H3K} 4$ (C) and histone acetylation at H3K4 (E) while suppressing histone trimethylation at H3K27 (D) at the HIV LTR. ChIP assay was performed using anti-H3K4Cr $(n=3)$, anti-H3K4Ac $(n=3)$, and anti-H3K27Me3 $(n=6)$ antibodies after J-Lat A1 cells were treated with $30 \mathrm{mM} \mathrm{Na}$-Cro for 4 hours. PCR primers were specific for the HIV LTR region. ${ }^{* *} P<0.01$ versus control treatment. (F) Reactivation of latent HIV was examined using different epigenetic modifiers. J-Lat A1 cells were treated with $30 \mathrm{mM} \mathrm{Na-Cro,} 500 \mathrm{nM}$ vorinostat, $2 \mu \mathrm{M}$ GSK343, or $5 \mu \mathrm{M}$ AZA-dC. HIV expression was measured by RT-qPCR. ${ }^{* *} P<0.01$ and ${ }^{* * *} P<0.001$ versus control treatment $(n=3)$. (G) Pretreatment with the ACSS2 inhibitor AR12 ( $5 \mu \mathrm{M}$ for 30 minutes) dampened histone crotonylation-induced reactivation of latent HIV. ${ }^{* *} P<0.001$ versus control treatment; ${ }^{\# \#} P<0.001$ versus $\mathrm{Na}$-Cro treatment $(n=4)$. (H) Suppression of ACSS2 by AR12 decreased histone crotonylation at the HIV LTR. J-Lat A1 cells were pretreated with $5 \mu$ M AR12 for 30 minutes, followed by Na-Cro (20 mM) addition and incubation for 4 hours. Crotonylation of H3K18Cr at the HIV LTR was measured by ChIP assay. ${ }^{* *} P<0.01$ versus control treatment; ${ }^{\# \#} P<0.01$ versus Na-Cro treatment $(n=3)$. The data were analyzed with a Student's $t$ test or 1 -way ANOVA.

expression of ACSS2 in the HIV LTR reporter cell line TZM-bl (a CXCR4-positive HeLa cell line engineered to express CD4 and CCR5 constitutively and firefly luciferase under the control of the HIV LTR). A significant reduction was detected in the HIV LTRdriven luciferase expression in the ACSS2 siRNA-treated TZM-bl cells as compared with the cells treated with control siRNA, with or without Na-Cro treatment (Figure 1, F-H). Collectively, our data demonstrate that induction of ACSS2 is effective in disrupting HIV latency across multiple models of HIV latency ex vivo and in vitro and crotonylation-mediated transcription of HIV was predominantly driven by ACSS2.

ACSS2 enzyme leads to chromatin reprogramming through histone crotonylation. To determine the molecular mechanism of ACSS2-induced reactivation of latent HIV, we examined several histone modifications in Na-Cro-treated $\mathrm{T}$ cells and assessed expression of major components of transcription factors involving HIV transcription and viral latency. Interestingly, induction of ACSS2 increased not only H3K 4 crotonylation (H3K4cr) but also H3K 4 acetylation (H3K4Ac) and H3K18 acetylation (H3K18Ac). In contrast, it markedly decreased H3K27 trimethylation (H3K27Me). Changes in histone crotonylation and acetylation were detectable even at low Na-Cro concentrations (Figure 2A). These data suggested that induction of ACSS2 following addition of Na-Cro leads to global reprogramming of histone tails in $\mathrm{CD} 4^{+} \mathrm{T}$ cells. However, it did not alter expression of transcription factors essential for HIV transcription except for a minimal change in the CDK9 protein levels (Figure 2B). Evaluation of chromatin remodeling in the HIV LTR by chromatin immunoprecipitation (ChIP) assay showed that induction of ACSS2 increased the crotonylation and acetylation of histone tail at $\mathrm{H} 3 \mathrm{~K} 4$ but decreased trimethylation of histone tail at H3K27 in the HIV LTR (Figure 2, C-E).

In agreement with our previous findings and other reports, we found that inhibition of histone methyltransferase (EZH2) by GSK343 or inhibition of DNA methyltransferase by 5 -aza-2' deoxycytidine (AZA-dC) minimally reactivated latent $\operatorname{HIV}(7,33)$. Induction of ACSS2 by Na-Cro or suppression of HDAC by vorinostat had similar effects in reactivating latent HIV in J-Lat A1 cells, and these findings were comparable to data from the resting $\mathrm{CD}^{+} \mathrm{T}$ cells from HIV-positive patients under ART (Figure 2F). To further verify the role of histone crotonylation in the maintenance of HIV latency, we pharmacologically suppressed the activity of ACSS2 using AR12, a known ACSS2 inhibitor, and investigated the 

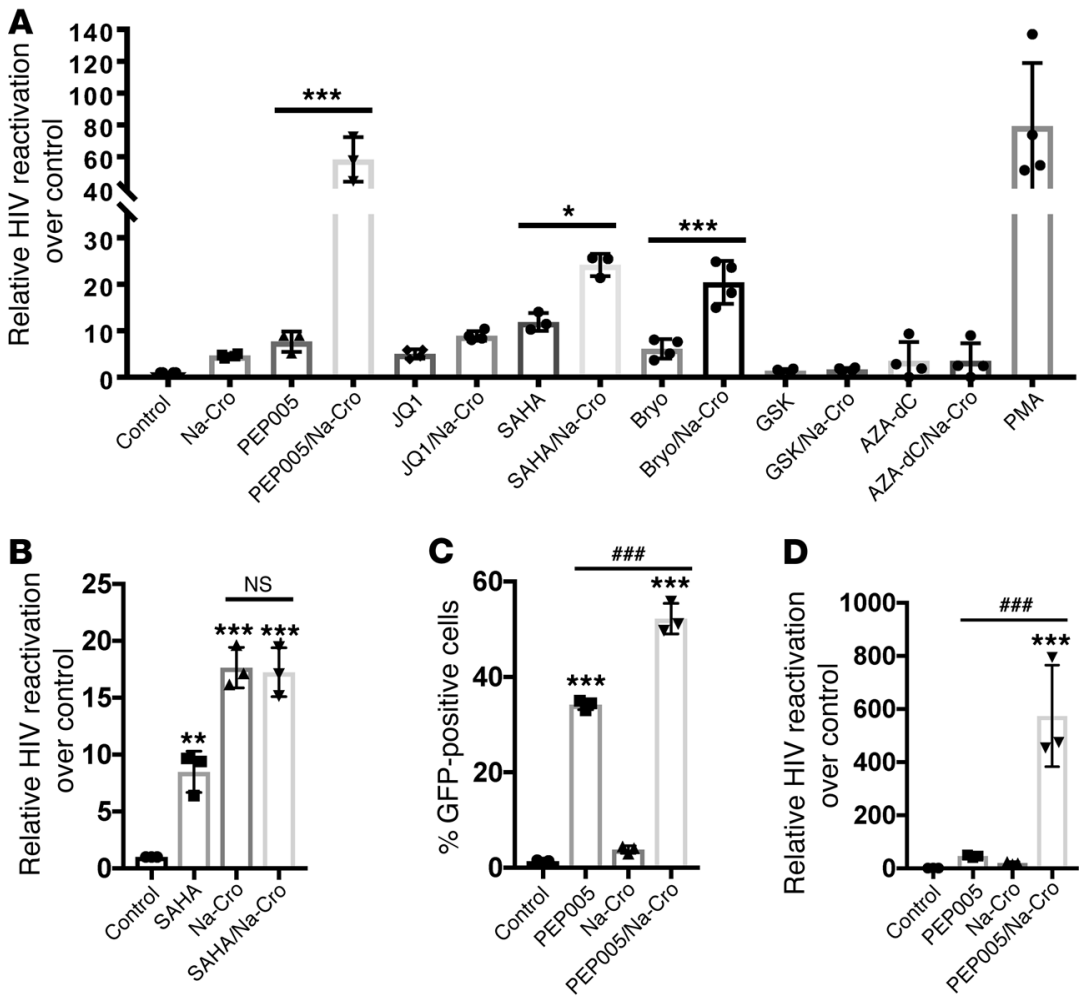

Figure 3. A synergistic reactivation of latent HIV expression by the histone crotonylation in combination with other LRAs. (A) The J-Lat A1 cells were treated overnight with $30 \mathrm{mM} \mathrm{Na}-\mathrm{Cro}(n=3), 6 \mathrm{nM}$ PEP005 ( $n=3), 2 \mu \mathrm{M}$ JQ1 $(n=4), 10$ nM bryostatin ( $n=$ 4), $250 \mathrm{nM}$ vorinostat $(n=3), 2 \mu \mathrm{M} \mathrm{CSK343}(n=4), 5$ $\mu \mathrm{M} \mathrm{AZA}-\mathrm{dC}(n=4)$, or $5 \mathrm{ng} / \mathrm{ml} \mathrm{PMA}(n=4)$ individually or in combination as indicated in the panel and evaluated for HIV reactivation by RT-qPCR. ${ }^{*} P<0.05$ and ${ }^{* * *} P<0.001$ versus LRA alone. (B) The J-Lat A1 cells were pretreated with $250 \mathrm{nM}$ of vorinostat; then 40 $\mathrm{mM}$ of $\mathrm{Na}$-Cro was added overnight. The HIV reactivation was measured by real-time PCR. ${ }^{* *} P<0.01$ and ${ }^{* *} P<0.001$ versus control treatment $(n=3)$. NS, not significant compared with $\mathrm{Na}-\mathrm{Cro}$ treatment alone. (C) Effect of $30 \mathrm{mM} \mathrm{Na}$-Cro, individually or in combination with PEP005, on reactivation of latent HIV was tested. The cells were treated overnight, and J-Lat A1 cells with HIV reactivation were measured by detection of GFP-expressing cells using flow cytometry. ${ }^{* *} P<$ 0.001 versus control treatment; \#\#\# $P<0.001$ versus PEP005 alone $(n=3)$. (D) U1 cells were treated similarly as in C; RT-qPCR was performed to measure viral expression. ${ }^{* *} P<0.001$ vs. untreated control; ${ }^{\# \# \#<}$ 0.001 versus PEP005 treatment alone $(n=3)$. The data were analyzed with 1-way ANOVA. downstream effects on HIV transcription (34). Induction of ACSS2 by $\mathrm{Na}$-Cro had 8-fold higher HIV reactivation compared with the untreated control. In contrast, addition of the ACSS2 inhibitor AR12 resulted in a substantial decrease in latent HIV reactivation (>60\%). Thus, pretreatment with AR12 diminished the effects of ACSS2 induction in disrupting HIV latency (Figure 2G). Importantly, pharmacologic inhibition of the ACSS2 enzyme markedly dampened histone crotonylation at HIV LTR (Figure 2H). Collectively, our data show that ACSS2-driven histone crotonylation at HIV LTR remodeled the histone tails to reactivate HIV from latency. These data identify a new mechanism that maintains HIV latency through limitation of ACSS2, thereby reducing histone crotonylation at the HIV LTR.

ACSS 2 reactivates HIV synergistically with other LRAs. The establishment and maintenance of HIV latency involve several molecular signaling pathways. Therefore, an efficient reactivation of latent HIV in the "shock-and-kill" approach may require the use of a combination of LRAs targeting different viral latency mechanisms. We sought to determine whether an intervention to induce histone crotonylation to reactivate latent HIV was synergistic with other LRAs that have different mechanisms of action. In combination treatments, J-Lat A1 cells were pretreated with Na-Cro, followed by treatment with other LRAs. Our data showed that crotonylation by Na-Cro addition was synergistic with PEPO05, bryostatin-1, or vorinostat in reactivating latent HIV. Combination with Na-Cro increased reactivation of latent HIV 8-fold, 3.3-fold, and 2.0-fold compared with single treatment of PEPO05, bryostatin-1, or vorinostat (Figure 3A and Supplemental Figure 2A). The protein kinase $\mathrm{C}$ (PKC) agonists PEP005 and bryostatin-1 have been shown to reactivate $\mathrm{HIV}$ through the $\mathrm{PKC} / \mathrm{NF}-\kappa \mathrm{B}$ pathway $(7,35)$. Vorinostat is an HDAC inhibitor that activates HIV expression through chro- matin remodeling by inhibiting histone deacetylation (12). A combination of $\mathrm{Na}$-Cro with the bromodomain protein BRD4 inhibitor JQ1, the histone methyltransferase inhibitor GSK343, or the DNA methyltransferase inhibitor AZA-dC $(7,36)$ did not show any synergistic effect on HIV reactivation in J-Lat A1 cell lines. It is intriguing to note that pretreatment with $\mathrm{Na}$-Cro followed by vorinostat treatment demonstrated a synergistic effect on HIV reactivation (Figure $3 \mathrm{~A})$. In contrast, pretreatment with vorinostat followed by $\mathrm{Na}-\mathrm{CrO}$ failed to show any combination effect in either J-Lat A1 cells or U1 cell models of HIV latency (Figure 3B and Supplemental Figure 2B). Since a combination of Na-Cro and PEPOO5 displayed the most potent effect on reactivation of latent HIV, we further sought to measure the magnitude of HIV LTR reactivation. A significant level of viral reactivation was detected by flow cytometric analysis (Figure 3C). Similarly, a synergistic increase in HIV reactivation was also identified in U1 monocytic cells (Figure 3D).

Induction of ACSS2 has no impact on immune activation. We examined whether induction of histone crotonylation in $\mathrm{T}$ cells impacted the level of immune activation in these cells. Peripheral blood mononuclear cells from HIV-negative healthy donors $(n=5)$ were treated with PMA/ionomycin or Na-Cro for 24 or 72 hours. Changes in the immune cell status were examined by measurement of the levels of HLA-DR, CD69, and PD-1 in the T cell subsets by flow cytometric analysis (Supplemental Figure 3). Among $\mathrm{CD}^{+}, \mathrm{CD}^{+}$, or $\mathrm{CD}^{+} \mathrm{T}$ cells, Na-Cro-induced histone crotonylation had no effect on the expression of those immune activation or checkpoint markers. In contrast, PMA/ionomycin treatment significantly induced CD69, HLA-DR, and/or PD-1 expression (Supplemental Figure 3, A-C). These findings suggest that histone crotonylation by induction of ACSS2 does not cause changes in $\mathrm{T}$ cell activation or suppression. 

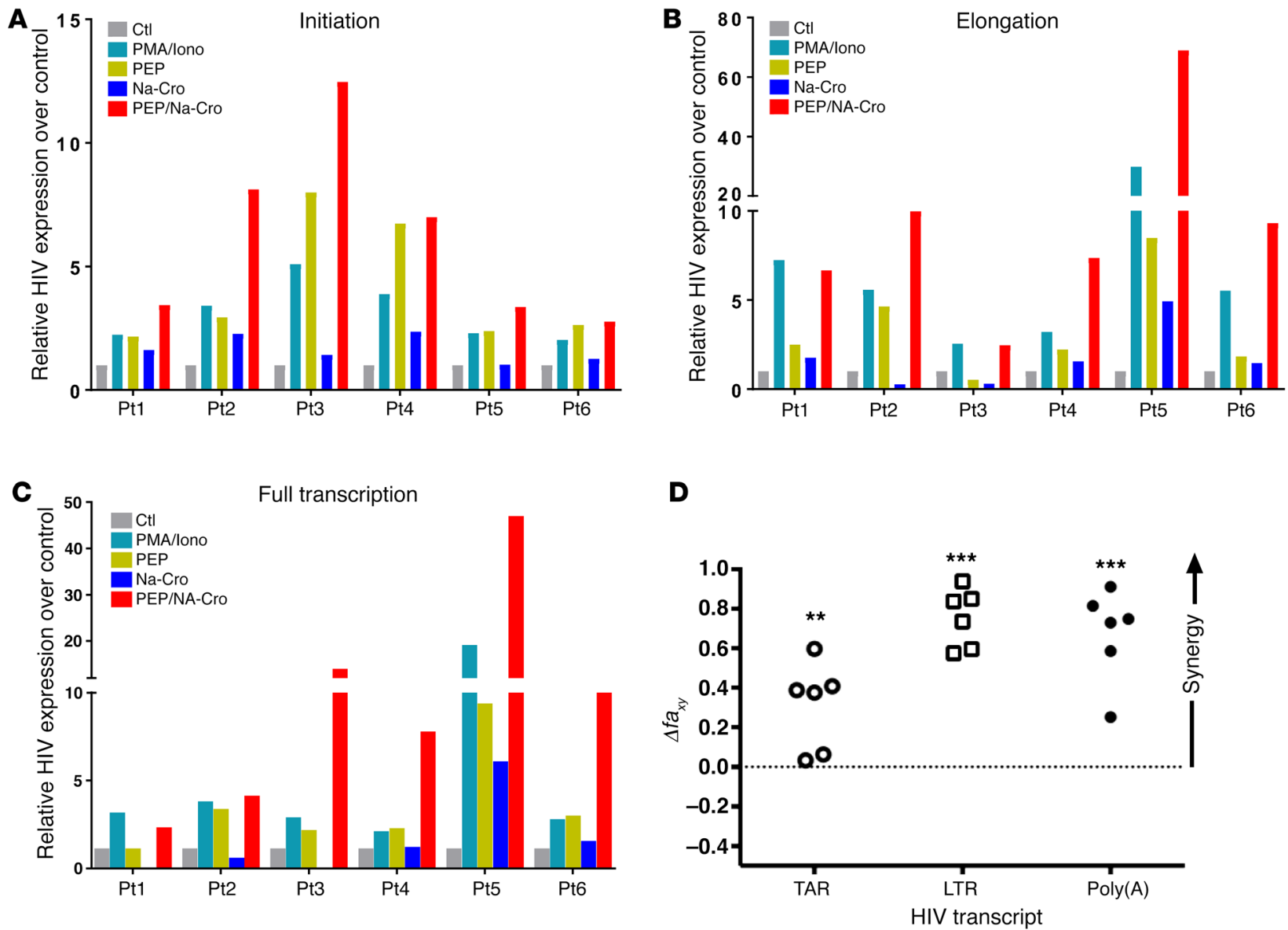

Figure 4. Histone crotonylation modifier and PEP005 synergistically reactivate latent HIV in CD4+ $\mathrm{T}$ cells from HIV-infected individuals under suppressive ART. (A-C) Primary CD4+ ${ }^{+}$cells isolated from HIV-positive patients $(n=6)$ under ART were treated with $100 \mathrm{ng} / \mathrm{ml} \mathrm{PMA} \mathrm{plus} 2 \mu \mathrm{M}$ ionomycin, $12 \mathrm{nM}$ PEP005, $40 \mathrm{mM} \mathrm{Na}$-Cro, or $12 \mathrm{nM}$ PEP005 combined with $40 \mathrm{mM} \mathrm{Na}$-Cro for 6 hours. Viral transcription from total RNA was analyzed by reverse transcriptase digital droplet PCR with primers targeting initiation (TAR region) (A), elongation (long LTR) (B), or full transcription [poly(A) region] (C) of the HIV genome. (D) Na-Cro synergistically reactivates the latent HIV with PEP005 in primary CD4+ ${ }^{+}$cells isolated from patients under suppressive ART. The difference between the observed fraction and predicted fraction or faxyo-faxyp $\left(\Delta f a_{x y}\right)$ was determined by Bliss independence analysis from 6 patient samples as described in the Supplemental Methods. ${ }^{* *} P<0.01$ and ${ }^{* *} P<0.001$ versus $f a_{x y p}(n=6)$.

Induction of histone crotonylation in combination with other LRAs disrupts HIV latency in primary $C D 4^{+} T$ cells ex vivo. In HIV latency cell culture models in vitro, we found that Na-Cro displayed the greatest synergy for HIV reactivation in combination with the PKC agonist PEPO05 as compared with other LRAs (Figure 3A and Supplemental Figure 2A). We sought to validate this finding in the ex vivo primary $\mathrm{CD}^{+} \mathrm{T}$ cells from $\mathrm{HIV}$-infected patients. Peripheral blood samples were obtained from $6 \mathrm{HIV}$ infected patients receiving suppressive ART. They had undetectable plasma viral loads ( $<20$ copies $/ \mathrm{ml}$ plasma) and greater than $400 \mathrm{CD}^{+} \mathrm{T}$ cell number per microliter of blood (465-885 cells $\left./ \mu \mathrm{l}\right)$ (Supplemental Table 1, patients 1-6). Primary CD4 ${ }^{+} \mathrm{T}$ cells were treated with PMA/ionomycin, PEP005, Na-Cro, or PEPO05 in combination with Na-Cro for 6 hours. HIV transcription following viral reactivation was measured by digital droplet PCR targeting the TAR region (initiation), long LTR region (proximal elongation), or poly(A) region (completed transcription) of the HIV genome $(7,37,38)$. In concordance with the findings from resting $\mathrm{CD}^{+}$ $\mathrm{T}$ cells using quantitative viral outgrowth assay ex vivo and from HIV latency cell models in vitro, histone crotonylation induced initiation of HIV transcripts in primary $\mathrm{CD}^{+} \mathrm{T}$ cells from 5 of 6 patients and elongation of HIV transcripts in 4 of 6 patients (Figure 4, A-C). Treatment with PEPO05 induced the initiation of HIV transcription in $\mathrm{CD}^{+} \mathrm{T}$ cells from all 6 patients, while the elongation of long viral transcripts was detected in 5 of 6 patient samples. In agreement with our previous reports, PEP005 alone also induced full-length transcription of HIV in most patient samples (5 of 6 samples) (7). These data demonstrate the ability of histone crotonylation by ACSS2 induction to reactivate HIV from latency in primary $\mathrm{CD}^{+} \mathrm{T}$ cells from HIV-infected patients under suppressive ART. A combination of ACSS2 induction with PEPOO5 in primary $\mathrm{CD}^{+} \mathrm{T}$ cells from HIV-infected patients induced greater reactivation of latent $\mathrm{HIV}$ than either agent alone (Figure $4, \mathrm{~A}-\mathrm{C}$ ) and exceeded levels induced even by PMA/ionomycin treatment in most of the patient samples, indicating a significant potency of this combination treatment (Supplemental Figure 4). An analysis of synergy using the Bliss independence model confirmed that the combination of PEPOO5 and Na-Cro synergistically reactivates latent HIV ex vivo (Figure 4D, Supplemental Figure 5, and ref. 39). These findings are in agreement with our data from HIV latency 
A

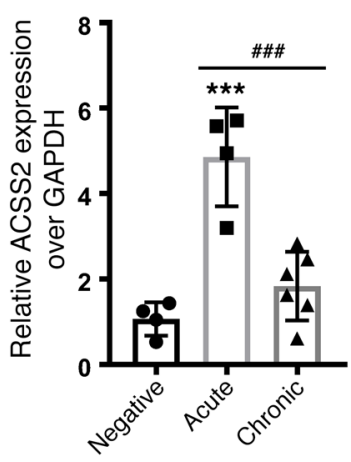

B

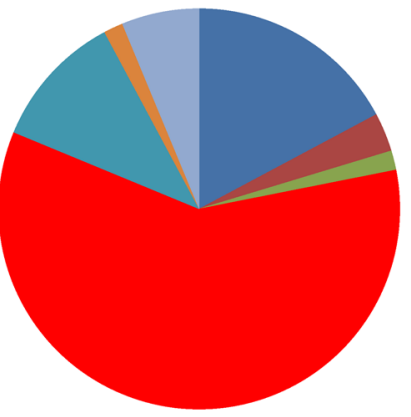

- Amino acid

Energy

- Nucleotide

- Xenobiotics
- Peptide

- Lipid metabolism

- Cofactors and vitamins
C

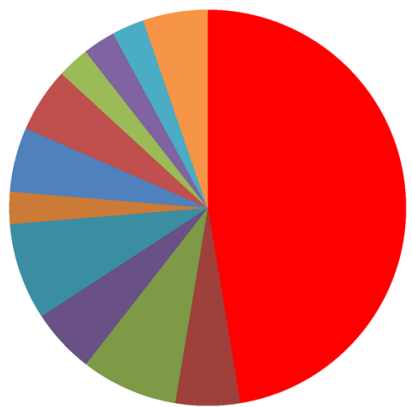

- Fatty acid metabolism

- Phosphatidylcholine

- Plasmalogen

- Monoacylglycerol

- Sphingolipid metabolism

- Mevalonate metabolism

- Phospholipid metabolism

- Lysophospholipid

- Lysoplasmalogen

- Diacylglyercol

- Ceramides

Corticosteroids
D

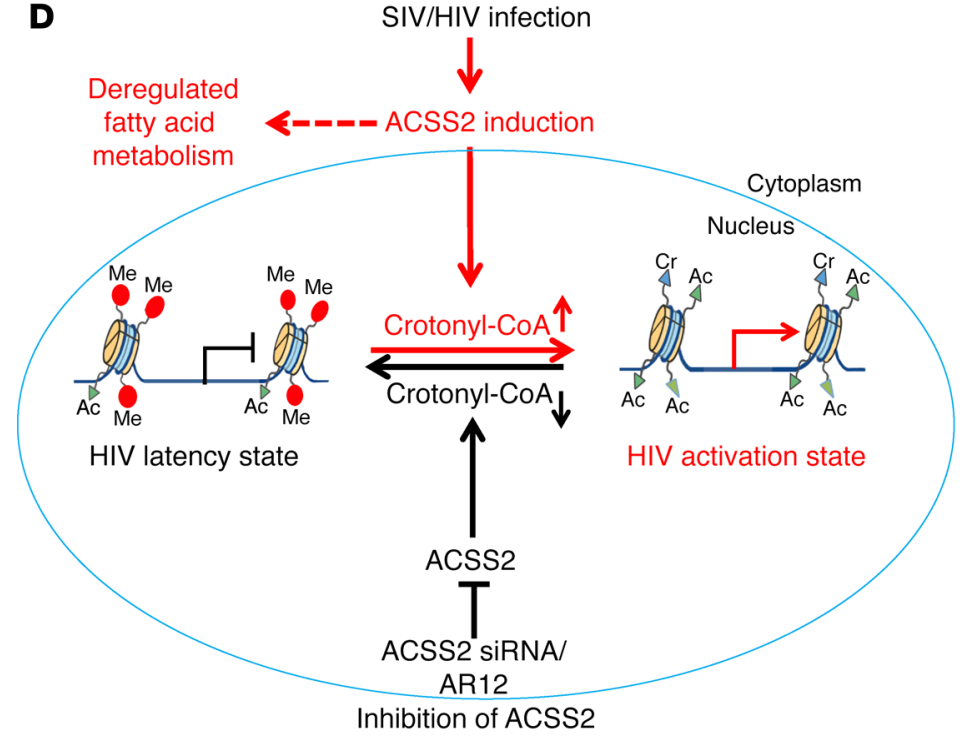

Figure 5. Early SIV infection induces expression of the fatty acid metabolic gene ACSS2. (A) Expression of ACSS2 was induced in the intestinal tissues during early SIV infection. RNA from control $(n=4)$, acutely SIV-infected ( $1-2$ weeks, $n=4$ ), and chronically SIV-infected ( $26-28$ weeks, $n=6$ ) rhesus macaques was extracted, and expression of ACSS2 was determined by RT-qPCR. ${ }^{* *} P<0.001$ versus negative infection; \#\#P<0.001 versus acute viral infection. The data were collected from 14 tissue samples and analyzed with 1-way ANOVA. (B and C) Profiles of lipid or fatty acid metabolism products during SIV infection. Metabolomic analysis was performed on the luminal contents from intestinal loops of 10 -week SIV-infected ( $n=9$ in 3 animals) or SIV-negative ( $n=9$ in 3 animals) rhesus macaques. (D) Viral infection or addition of crotonyl-CoA induces the expression of the fatty acid metabolic enzyme ACSS2, leading to epigenetic regulation of HIV latency/transcription by histone crotonylation. Suppression of ACSS2 by siRNA or AR12 inhibits HIV replication, which may facilitate the establishment of HIV latency. cell culture models in vitro (Figure 3). While the combination of $\mathrm{Na}$-Cro with vorinostat (SAHA) increased reactivation of latent HIV compared with vorinostat alone in 4 of 6 primary $\mathrm{CD}^{+}{ }^{+} \mathrm{T}$ cell samples isolated from patients under suppressive ART, synergy was not observed (Supplemental Figure 6). Therefore, the impact of crotonylation on acetylation-induced latent HIV reactivation warrants further investigation. Collectively, our data suggest that histone crotonylation by ACSS2 induction is not only able to reactivate the latent $\mathrm{HIV}$ in primary $\mathrm{CD}^{+} \mathrm{T}$ cells from HIV-infected patients under ART but demonstrates synergism with PEPO05, an LRA targeting $\mathrm{PKC} / \mathrm{NF}-\kappa \mathrm{B}$ signaling, in its reactivation activity.

ACSS 2 is induced during acute SIV infection in the rhesus macaque model of AIDS in vivo. As shown above, the induction of ACSS2 results in chromatin remodeling via crotonylation of histone tails at the HIV LTR; the latter drives the replication of HIV or reactivates HIV from latency in vitro and ex vivo. To determine whether viral infection affects ACSS2 expression in vivo, we examined the expression of ACSS2 in intestinal tissues during viral infection in the SIV-infected rhesus macaque model of AIDS (Supplemental Table 2). We found that ACSS2 expression was highly induced in the intestine during the primary acute SIV infection in vivo. How- ever, the ACSS2 levels decreased during the chronic stage of viral infection (Figure 5A). These findings suggest that ACSS2 may influence viral replication in vivo. Interestingly, ACSS2 is one of the essential enzymes for controlling lipid or fatty acid metabolism $(19,40)$. To determine whether lipid or fatty acid metabolism was altered during viral infection, we analyzed the metabolic profiles of the luminal contents from SIV-infected rhesus macaques. We found that approximately $60 \%$ of the changes in the metabolic products during SIV infection were related to lipid metabolism. Within lipid metabolism, approximately $45 \%$ of the altered metabolic products belonged to fatty acid metabolism (Figure 5, B and C). These data indicate that SIV/HIV infection-modulated fatty acid metabolism may be potentially involved in the regulation of HIV replication through induction of ACSS2 in the gut during viral infection in vivo.

\section{Discussion}

Latent HIV reservoirs in the host are established very early in viral infection (41-45). Since HIV provirus preferentially integrates in transcriptionally active regions of the host genome (8), it is necessary to establish a quiescent chromatin microenvironment and 
interactions among cellular and viral transcriptional regulators for inducing and maintaining HIV latency $(9,46)$. Latent HIV is reactivated by interference with chromatin modifications as evidenced by efficacy of HDAC inhibitors or EZH2 inhibitors in reactivation of latent HIV (16). However, the level of latent HIV reactivation is relatively modest in $\mathrm{CD} 4^{+} \mathrm{T}$ cell cultures in vitro and in ex vivo primary $\mathrm{CD} 4^{+} \mathrm{T}$ cells from HIV-infected patients $(7,15,47-49)$. It is possible that HIV latency is regulated by additional mechanisms of histone modifications that have yet to be discovered. After inducing ACSS2 and histone crotonylation, the ability of the HDAC inhibitor vorinostat to reactivate latent HIV was significantly enhanced, indicating that an efficient reactivation of latent HIV by HDAC inhibitors may require the crotonylation of histone tails at the HIV LTR. Our findings point to the need to further characterize the epigenetic landscape of the HIV LTR and investigate multiple epigenetic modifications to achieve optimal viral transcription or silencing. Previous studies reported the ability of SIRT1 and SIRT3 proteins from the sirtuin (SIRT) family to erase crotonylation marks from lysine residues in $\mathrm{HeLa}$ S3 cell lines (22). However, our data did not support an essential role of SIRT1 or SIRT3 as a decrotonylase at the HIV LTR and suggest that regulation of HIV latency is independent of sirtuins (data not shown).

We and others have previously reported that the lipid metabolism was altered in HIV-infected primary $\mathrm{CD} 4^{+} \mathrm{T}$ cells in vitro or in the intestine during early stages of HIV infection in vivo (27-29). We also found that expression of the fatty acid enzyme ACSS2 was significantly induced in HIV-infected individuals following therapy interruption (50). In the current study, we have identified the modulation of fatty acid metabolism and the induction of ACSS2 expression in intestinal tissues during early stages of SIV infection in vivo. Aberrant fatty acid metabolism may activate proinflammatory signaling and disrupt mucosal integrity (51). It is not known whether host metabolites produced during HIV/SIV infection are responsible for gut mucosal damage in vivo, which is under investigation. Further analysis showed that ACSS2 can also influence HIV replication and viral latency by modulating histone crotonylation at HIV LTR. Disruption of HIV latency was observed by histone crotonylation following the induction of ACSS2 in vitro and ex vivo. Therefore, our findings favor a model whereby suppression of histone crotonylation inhibits reactivation of latent HIV and supports the maintenance of viral latency (Figure 5D). Previously, we found that expression of the ACSS2 gene was significantly induced in the intestinal biopsies of HIV-infected patients following interruption of ART in vivo (50). Our data, for the first time to our knowledge, have linked fatty acid metabolism to the epigenetic regulation of HIV transcription and maintenance of viral latency through crotonylation of histone tails by ACSS2 at the HIV LTR.

HIV RNA transcription can be detected in the absence of the production of virus and viral antigens and therefore may be insufficient to trigger viral cytolytic effects or immune recognition and clearance of reservoir cells $(1,52)$. Recently, Pollack et al. reported that even defective viruses can produce viral proteins that can be recognized by cytotoxic T lymphocytes (CTLs) mediating immune clearance (53). Detection of polyadenylated HIV RNA is considered to be the best correlate of viral production (39). In this study, we assessed HIV reactivation by measuring HIV transcripts representing the viral initiation (TAR), elongation (long LTR), and complete transcription (polyadenylated RNA). In addition, we also measured HIV p24 levels in culture supernatants as a correlate of viral production. When induction of ACSS2 was used alone to reverse HIV latency, the magnitude of effects on initiation of transcription was greater than that of effects on the elongation or generation of fully transcribed HIV RNA. However, detection of HIV p24 outgrowth from the resting $\mathrm{CD} 4^{+} \mathrm{T}$ cells in quantitative viral outgrowth assay indicated that the net effects of ACSS2-induced HIV transcription were sufficient to elicit production of viral p24 and virus. Importantly, our data show a remarkable synergistic reactivation of latent $\mathrm{HIV}$ when histone crotonylation is combined with the PKC agonist PEP005, vorinostat, or JQ1 in T cell cultures in vitro and/or $\mathrm{CD}^{+}{ }^{+} \mathrm{T}$ cells from HIV-infected patients ex vivo. A robust reactivation of latent HIV following the combination treatment of cells from HIV-infected patients indicated that increased levels of viral particles could be induced that are recognized by CTLs for immune clearance when an effective killing strategy is applied. On the other hand, inhibition of histone crotonylation by suppression of the crotonyl-CoA-converting enzyme ACSS2 dampened latent HIV reactivation, indicating that the loss of ACSS2 leads to suppression of HIV transcription and a potential role of histone decrotonylation in establishment of HIV latency. SIRT proteins were characterized as decrotonylases $(22,54)$. However, our data did not support their role as histone decrotonylases in the HIV LTR. In contrast to many if not most other LRAs, induction of ACSS2 by Na-Cro did not induce immune activation or modulate levels of the immune checkpoint protein PD-1 on $\mathrm{CD}^{+}$ $\mathrm{T}$ cells or $\mathrm{CD} 8^{+} \mathrm{T}$ cells.

In summary, our data uncovered an important role for the fatty acid metabolic enzyme ACSS2 in the regulation of HIV transcription by crotonylation of histone tails at the HIV LTR. We have identified decrotonylation of histone tails at the HIV LTR as a potential novel histone mark for establishment and maintenance of HIV latency. This epigenetic modification mechanism and its reversal open new avenues for HIV cure approaches.

\section{Methods}

Further information can be found in Supplemental Methods, available online with this article.

Cell culture. J-Lat A1 cells (derived from Jurkat cells harboring HIV LTR-Tat-GFP gene) and U1 cells (promonocytic cell line harboring HIV proviruses with defective Tat gene) were obtained from the NIH AIDS Reagent Program and cultured at $37^{\circ} \mathrm{C}$ with $5 \% \mathrm{CO}_{2}$ in RPMI 1640 medium containing $10 \% \mathrm{FBS}$ and $1 \%$ penicillin/streptomycin as previously described (7).

Latency reversal agents. For reactivation of latent HIV, cells were treated with sodium crotonate (Na-Cro; Sigma-Aldrich), PEPOO5 (Tocris Bioscience), vorinostat (Santa Cruz Biotechnology), GSK343 (Sigma-Aldrich), or AZA-dC (Santa Cruz Biotechnology) for 4, 18, or 24 hours. A combination treatment involved pretreatment of cells with Na-Cro for 4 hours, followed by treatment with PEP005, bryostatin-1 (Calbiochem), JQ1 (Biovision), vorinostat, GSK343, or AZA-dC for 18 hours. For inhibiting acyl-CoA synthetase 2 enzyme (ACSS2) and crotonylation, cells were pretreated with the ACSS2 inhibitor AR12 (Selleckchem) for 30 minutes, followed by Na-Cro treatment for 4 or 18 hours. 
Gene knockdown by ACSS2 siRNA. TZM-bl HIV transcription/ replication reporter cells $\left(1 \times 10^{5}\right)$ were seeded in the 12 -well plate, and then the cells were transfected with ACSS2 siRNA (M-010396) or nontargeting control siRNA (M-006526) (Dharmacon) twice. The cells were treated with or without $40 \mathrm{mM} \mathrm{Na}$-Cro overnight and collected for luciferase assay of HIV transcription and/or lysed for quantitative reverse transcriptase PCR (RT-qPCR) of ACSS2 gene expression.

Primary $C D 4^{+} T$ cell isolation, treatment, and digital droplet $P C R$ assays. Peripheral blood samples were obtained from HIV-infected individuals (age ranging from 22 to 62 years) on suppressive ART for more than 2 years ( $n=12$, average age $10.6 \pm 5.7$ years). The plasma viral loads were below the detection level ( $<20$ copies $/ \mathrm{ml}$ plasma), and the average $\mathrm{CD} 4^{+} \mathrm{T}$ cell number was $680.2 \pm 225.6$ cells $/ \mu \mathrm{l}$. The primary $\mathrm{CD}^{+} \mathrm{T}$ cells were isolated using the EasySep kit (STEMCELL Technologies) as previously described $(7,15)$. The purified $\mathrm{CD} 4^{+} \mathrm{T}$ cells were plated at a density of $0.5 \times 10^{6}$ to $1 \times 10^{6}$ cells and treated with DMSO, $200 \mathrm{ng} / \mathrm{ml}$ PMA plus $2 \mu \mathrm{M}$ ionomycin, $12 \mathrm{nM}$ PEPO05, $40 \mathrm{mM}$ Na-Cro, or $12 \mathrm{nM}$ PEPO05 plus $40 \mathrm{mM}$ Na-Cro for 6 hours. Cell pellets were collected for RNA isolation. Initiation, elongation, or full transcription of HIV was analyzed with digital droplet PCR assays as reported before $(7,37,38)$.

Quantitative viral outgrowth assay. Peripheral blood mononuclear cells (PBMCs) were obtained from HIV-infected individuals on suppressive ART $(n=5)$ by continuous-flow leukapheresis. Isolation of resting $\mathrm{CD}^{+} \mathrm{T}$ cells and quantification of replication-competent virus were performed as previously described (41). Briefly, approximately 34 million to 50 million resting $\mathrm{CD}^{+} \mathrm{T}$ cells per each treatment condition were plated in replicate limiting dilutions of 2.5 million (18 cultures), 0.5 million ( 6 cultures), and 0.1 million ( 6 cultures) cells per well and stimulated for 24 hours with either (a) PHA (Remel, Thermo Fisher Scientific), a 5-fold excess of allogeneic irradiated PBMCs from a seronegative donor, and IL-2; (b) $40 \mathrm{mM} \mathrm{Na-Cro} \mathrm{plus} \mathrm{IL-2} \mathrm{or} 350$ nM SAHA plus IL-2; or (c) IL-2 as unstimulated control. Cultures were washed and cocultivated with $\mathrm{CD} 8^{+} \mathrm{T}$ cell-depleted PBMCs that were obtained from selected HIV-seronegative donors previously screened for adequate CCR5 expression. Culture supernatants were harvested on days 15 and 19 and assayed for virus production by $\mathrm{p} 24$ antigen capture ELISA (ABL). Cultures were scored as positive if $\mathrm{p} 24$ was detected at day 15 and was increased in concentration at day 19. The number of resting $\mathrm{CD} 4^{+} \mathrm{T}$ cells in infected units per million was estimated by a maximum likelihood method (55).

Statistics. Data represent the mean \pm SEM, calculated using all data points from at least 3 independent experiments. Statistical significance was determined using a 2-way Student's $t$ test for samples with only 2 groups. For multiple-comparison analysis of samples from 3 or more groups, we applied 1-way ANOVA analysis followed by a post-hoc Tukey's test, and a $P$ value of less than 0.05 was considered significant.

Study approval. This study was carried out under the recommendations of the Public Health Service Policy on Humane Care and Use of Laboratory Animals. Human peripheral blood samples $(n=17)$ were obtained under informed written consent and a protocol approved by the Institutional Review Boards at UCD and the University of North Carolina at Chapel Hill. Animals were housed at the California National Primate Research Center at UCD, and procedures were approved by the Institutional Animal Care and Use Committee of UCD.

\section{Author contributions}

GJ and SD conceived and designed the experiments. GJ, DN, YT, MME, NMA, and GML performed the experiments. GJ, SAY, JKW, NMA, DMM, DJHO, and SD analyzed the data. NMA and GRT coordinated patient samples. GJ and SD wrote the manuscript.

\section{Acknowledgments}

We thank Lauren A. Hirao and Clarissa Santos Rocha for the initiation of the metabolomic study and Peggy Kim for technical support. We are very grateful to all patients for participating in this study. This work was supported by NIH grants AI123105 and AI43274 and a UC Davis Research Investments in Science and Engineering grant to SD; NIH grants U19-AI096113, UM1-AI126619, and UNC CFAR P30-AI504100 to DMM; and NIH grants AI116342, AI116218, DK108349, and AI132128 to JKW and SAY.

Address correspondence to: Satya Dandekar, Department of Medical Microbiology and Immunology, UCD, One Shields Drive, 3146 Tupper Hall, Davis, California 95616, USA. Phone: 530.752.3409; Email: sdandekar@ucdavis.edu.
1. Bruner KM, Hosmane NN, Siliciano RF. Towards an HIV-1 cure: measuring the latent reservoir. Trends Microbiol. 2015;23(4):192-203.

2. Margolis DM. How might we cure HIV? Curr Infect Dis Rep. 2014;16(3):392.

3. Siliciano JD, et al. Long-term follow-up studies confirm the stability of the latent reservoir for HIV-1 in resting CD $4{ }^{+}$T cells. Nat Med. 2003;9(6):727-728.

4. Finzi D, et al. Identification of a reservoir for HIV-1 in patients on highly active antiretroviral therapy. Science. 1997;278(5341):1295-1300.

5 . Wong JK, et al. Recovery of replication-competent HIV despite prolonged suppression of plasma viremia. Science. 1997;278(5341):1291-1295.

6. Chun TW, et al. Presence of an inducible HIV-1 latent reservoir during highly active antiretroviral therapy. Proc Natl Acad Sci U S A. 1997;94(24):13193-13197.

7. Jiang G, et al. Synergistic reactivation of latent
HIV expression by ingenol-3-angelate, PEP005, targeted NF-kB signaling in combination with JQ1 induced p-TEFb activation. PLoS Pathog. 2015;11(7):e1005066.

8. Hakre S, Chavez L, Shirakawa K, Verdin E. Epigenetic regulation of HIV latency. Curr Opin HIV AIDS. 2011;6(1):19-24.

9. Jiang G, Espeseth A, Hazuda DJ, Margolis DM. c-Myc and Sp1 contribute to proviral latency by recruiting histone deacetylase 1 to the human immunodeficiency virus type 1 promoter. J Virol. 2007;81(20):10914-10923.

10. du Chéné I, et al. Suv39H1 and HP1 $\gamma$ are responsible for chromatin-mediated HIV-1 transcriptional silencing and post-integration latency. EMBO J. 2007;26(2):424-435.

11. Friedman J, et al. Epigenetic silencing of HIV-1 by the histone $\mathrm{H} 3$ lysine 27 methyltransferase enhancer of Zeste 2. J Virol. 2011; 85(17):9078-9089.
12. Archin NM, et al. Administration of vorinostat disrupts HIV-1 latency in patients on antiretroviral therapy. Nature. 2012;487(7408):482-485.

13. Rasmussen TA, et al. Panobinostat, a histone deacetylase inhibitor, for latent-virus reactivation in HIV-infected patients on suppressive antiretroviral therapy: a phase $1 / 2$, single group, clinical trial. Lancet HIV . 2014;1(1):e13-e21.

14. Søgaard OS, et al. The depsipeptide romidepsin reverses HIV-1 latency in vivo. PLoS Pathog. 2015;11(9):e1005142.

15. Jiang $\mathrm{G}$, et al. Reactivation of HIV latency by a newly modified Ingenol derivative via protein kinase $\mathrm{C} \delta$-NF- $\mathrm{NB}$ signaling. AIDS. 2014;28(11):1555-1566.

16. Jiang G, Dandekar S. Targeting NF- $\kappa \mathrm{B}$ signaling with protein kinase $\mathrm{C}$ agonists as an emerging strategy for combating HIV latency. AIDS Res Hum Retroviruses. 2015;31(1):4-12.

17. Tan $\mathrm{M}$, et al. Identification of 67 histone marks and 
histone lysine crotonylation as a new type of histone modification. Cell. 2011;146(6):1016-1028.

18. Sabari BR, et al. Intracellular crotonyl-CoA stimulates transcription through p300-catalyzed histone crotonylation. Mol Cell. 2015;58(2):203-215.

19. Sabari BR, Zhang D, Allis CD, Zhao Y. Metabolic regulation of gene expression through histone acylations. Nat Rev Mol Cell Biol. 2017;18(2):90-101.

20. Li Y, Zhao D, Chen Z, Li H. YEATS domain: linking histone crotonylation to gene regulation. Transcription. 2017;8(1):9-14.

21. Andrews FH, et al. The Taf14 YEATS domain is a reader of histone crotonylation. Nat Chem Biol. 2016;12(6):396-398.

22. Bao X, et al. Identification of 'erasers' for lysine crotonylated histone marks using a chemical proteomics approach. Elife. 2014;3:e02999.

23. Abumrad NA, Davidson NO. Role of the gut in lipid homeostasis. Physiol Rev. 2012;92(3):1061-1085.

24. Stanley TL, Grinspoon SK. Body composition and metabolic changes in HIV-infected patients. J Infect Dis. 2012;205(suppl 3):S383-S390.

25. Bociąga-Jasik M, et al. Metabolic complications and selected cytokines in HIV-infected individuals. Pol Arch Med Wewn. 2014;124(1-2):27-35.

26. Koethe JR, Hulgan T, Niswender K. Adipose tissue and immune function: a review of evidence relevant to HIV infection. J Infect Dis. 2013;208(8):1194-1201.

27. Sankaran S, et al. Rapid onset of intestinal epithelial barrier dysfunction in primary human immunodeficiency virus infection is driven by an imbalance between immune response and mucosal repair and regeneration. JVirol. 2008;82(1):538-545.

28. Wu JQ, Dwyer DE, Dyer WB, Yang YH, Wang B, Saksena NK. Genome-wide analysis of primary $\mathrm{CD}^{+}$and $\mathrm{CD}^{+}{ }^{+} \mathrm{T}$ cell transcriptomes shows evidence for a network of enriched pathways associated with HIV disease. Retrovirology. 2011;8:18.

29. Guadalupe M, et al. Viral suppression and immune restoration in the gastrointestinal mucosa of human immunodeficiency virus type 1-infected patients initiating therapy during primary or chronic infection. JVirol. 2006;80(16):8236-8247.

30. Waagsbø B, et al. Low levels of short- and medium-chain acylcarnitines in HIV-infected patients. Eur JClin Invest. 2016;46(5):408-417.

31. Babirekere-Iriso $\mathrm{E}$, et al. Essential fatty acid composition and correlates in children with severe acute malnutrition. Clin Nutr ESPEN. 2016;11:e40-e46.

32. Nyirenda CK, et al. Plasma fatty acids in Zambian adults with HIV/AIDS: relation to dietary intake and cardiovascular risk factors. J Nutr Metab. 2015;2015:635817.

33. Kauder SE, Bosque A, Lindqvist A, Planelles $\mathrm{V}$, Verdin E. Epigenetic regulation of HIV-1 latency by cytosine methylation. PLoS Pathog. 2009;5(6):e1000495.

34. Koselny K, et al. Antitumor/antifungal celecoxib derivative AR-12 is a non-nucleoside inhibitor of the ANL-family adenylating enzyme acetyl CoA synthetase. ACS Infect Dis. 2016;2(4):268-280.

35. Díaz L, et al. Bryostatin activates HIV-1 latent expression in human astrocytes through a PKC and NF-kB-dependent mechanism. Sci Rep. 2015;5:12442.

36. Banerjee C, et al. BET bromodomain inhibition as a novel strategy for reactivation of HIV-1. JLeukoc Biol. 2012;92(6):1147-1154.

37. Kaiser $\mathrm{P}$, et al. Assays for precise quantification of total (including short) and elongated HIV-1 transcripts. J Virol Methods. 2017;242:1-8.

38. Shan L, et al. A novel PCR assay for quantification of HIV-1 RNA. J Virol. 2013;87(11):6521-6525.

39. Laird GM, et al. Ex vivo analysis identifies effective HIV-1 latency-reversing drug combinations. JClin Invest. 2015;125(5):1901-1912.

40. Xu H, et al. Acyl-CoA synthetase short-chain family member 2 (ACSS2) is regulated by SREBP- 1 and plays a role in fatty acid synthesis in caprine mammary epithelial cells. J Cell Physiol. 2018;233(2):1005-1016

41. Dandekar S. Pathogenesis of HIV in the gastrointestinal tract. Curr HIV/AIDS Rep. 2007;4(1):10-15.

42. Lackner AA, Lederman MM, Rodriguez B. HIV pathogenesis: the host. Cold Spring Harb Perspect Med. 2012;2(9):a007005.

43. Somsouk M, et al. Gut epithelial barrier and systemic inflammation during chronic HIV infection. AIDS. 2015;29(1):43-51.
44. Hirao LA, et al. Early mucosal sensing of SIV infection by paneth cells induces IL- $1 \beta$ production and initiates gut epithelial disruption. PLoS Pathog. 2014;10(8):e1004311.

45. Whitney JB, et al. Rapid seeding of the viral reservoir prior to SIV viraemia in rhesus monkeys. Nature. 2014;512(7512):74-77.

46. Tyagi M, Karn J. CBF-1 promotes transcriptional silencing during the establishment of HIV-1 latency. $Е M B O ~ J .2007 ; 26(24): 4985-4995$.

47. Klase Z, et al. Activation of HIV-1 from latent infection via synergy of RUNX1 inhibitor Ro5-3335 and SAHA. PLoS Pathog. 2014;10(3):e1003997.

48. Pandeló José D, et al. Reactivation of latent HIV-1 by new semi-synthetic ingenol esters. Virology. 2014;462-463:328-339.

49. Bouchat $S$, et al. Sequential treatment with 5-aza-2'-deoxycytidine and deacetylase inhibitors reactivates HIV-1. EMBO Mol Med. 2016;8(2):117-138.

50. Lerner $\mathrm{P}$, et al. The gut mucosal viral reservoir in HIV-infected patients is not the major source of rebound plasma viremia following interruption of highly active antiretroviral therapy. J Virol. 2011;85(10):4772-4782.

51. Rutkowsky JM, et al. Acylcarnitines activate proinflammatory signaling pathways. Am J Physiol Endocrinol Metab. 2014;306(12):E1378-E1387.

52. Bullen CK, Laird GM, Durand CM, Siliciano JD, Siliciano RF. New ex vivo approaches distinguish effective and ineffective single agents for reversing HIV-1 latency in vivo. Nat Med. 2014;20(4):425-429.

53. Pollack RA, et al. Defective HIV-1 proviruses are expressed and can be recognized by cytotoxic T lymphocytes, which shape the proviral landscape. Cell Host Microbe. 2017;21(4):494-506.e4.

54. Feldman JL, Baeza J, Denu JM. Activation of the protein deacetylase SIRT 6 by long-chain fatty acids and widespread deacylation by mammalian sirtuins. J Biol Chem. 2013;288(43):31350-31356.

55. Archin NM, Keedy KS, Espeseth A, Dang H, Hazuda DJ, Margolis DM. Expression of latent human immunodeficiency type 1 is induced by novel and selective histone deacetylase inhibitors. AIDS. 2009;23(14):1799-1806. 